\title{
SNIP: A Sensor Node-Initiated Probing Mechanism for Opportunistic Data Collection in Sparse Wireless Sensor Networks
}

\author{
Xiuchao Wu, Kenneth N. Brown, and Cormac J. Sreenan \\ Department of Computer Science, University College Cork, Ireland \\ Email:\{x.wu, k.brown, cjs\}@cs.ucc.ie
}

\begin{abstract}
In many potential wireless sensor network applications, the cost of the base station infrastructure can be prohibitive. Instead, we consider the opportunistic use of mobile devices carried by people in daily life to collect sensor data. As the movement of these mobile nodes is by definition uncontrolled, contact probing is a challenging task, particularly for sensor nodes which need to be duty-cycled to achieve long life.

We propose a Sensor Node-Initiated Probing mechanism for improving the contact capacity when the duty cycle of a sensor node is fixed. In contrast to existing mobile node-initiated probing mechanisms, in which the mobile node broadcasts a beacon periodically, in SNIP the sensor node broadcasts a beacon each time its radio is turned on according to its duty cycle. We study SNIP through both analysis and network simulation. The evaluation results indicate that SNIP performs much better than mobile-initiated probing. When the fixed duty cycle is lower than $1 \%$, the probed contact capacity can be increased by an order of 2-10; alternatively, SNIP can achieve the same amount of probed contact capacity with much less energy consumption.
\end{abstract}

\section{INTRODUCTION}

As wireless sensor networks mature, we expect to see longterm deployments for applications such as environmental monitoring, house water/gas/electricity meter reading, and structural health monitoring. These applications typically involve large numbers of sparsely deployed (static) sensor nodes that report data that is inherently delay tolerant, since the response (if any) requires human intervention over long time scales. For example, analysis of environmental monitoring data is rarely urgent, and meter readings for billing purposes can be delayed by weeks. Neighboring nodes in these sparse wireless sensor networks are far away from each other, and typically cannot communicate directly or even indirectly through multihop paths. On the other hand, deploying large numbers of fixed sink nodes would incur prohibitive costs in terms of deployment, maintenance, and data back-haul.

In [1][2], the use of resource-rich mobile nodes was proposed to move around in the deployed area and collect data from sensor nodes. Depending on the application, the mobile nodes can be either part of the external environment or part of the network, and their mobility can be either controllable or not. In this paper, we assume that mobility is not controlled and thus the sensed data is collected opportunistically. Mobile nodes could be specific devices carried by objects (animals, employees, etc.) who move around the deployed area for purposes other than data collection. More interestingly, they could also be smart phones and/or PDAs (installed with the corresponding radio and software) carried by unrelated people who pass through the deployed area in their daily life. Except the benefits of adopting mobile sinks discussed in [1][2], the cost of data collection can also be reduced significantly through exploiting the uncontrollable, but free mobility. Although opportunistic data collection may increase the data delivery latency [1], there are many promising wireless sensor network applications which are delay-tolerant and it is worthwhile to improve the performance of opportunistic data collection.

In the context of opportunistic data collection, the sensed data can be collected from a sensor node only after a mobile node approaches and they become aware of each other. Here, the event of the mobile node encountering with a sensor node is referred to as a contact. As the movement of these mobile nodes is uncontrollable, contact probing becomes a challenging task for sensor nodes which need to be duty-cycled to achieve a long life.

In this paper, we investigate the ways that sensor nodes and mobile nodes carry out contact probing and propose a Sensor Node-Initiated Probing mechanism for improving the probed contact capacity when the duty-cycle of a sensor node is fixed. SNIP is designed based on the following two reasonable assumptions, i.e., the radio of mobile nodes, which have relatively abundant energy via a re-chargable battery, can be always turned on and the radio of sensor nodes consumes almost the same amount of energy in transmitting and receiving/listening modes [3][4]. The basic principle of operation is that the sensor node initiates probing rather than a mobile node. Thus a sensor node must broadcast a beacon immediately after its radio is turned on.

SNIP has been studied through both analysis and simulation. The relationship between $\Upsilon$ (percent of the probed contact capacity), $d$ (sensor node's duty-cycle), and $T_{\text {contact }}$ (the length of a contact) has been modeled for SNIP. SNIP is also implemented in Contiki-OS [5] and extensive simulations are carried out using COOJA[6]. Both the analysis and simulation results indicate that SNIP outperforms mobile node-initiated probing mechanisms, and we quantify the impact of key parameters. A key conclusion is that with a sensor node duty- 
cycle that is lower than $1 \%$, the probed contact capacity can be increased by a factor of 2-10; alternatively, for probing the same amount of contact capacity, the energy consumed by SNIP is much less than the energy consumed by mobile node-initiated probing mechanisms.

This paper is organized as follows. Section II first investigates contact probing issues under the scenario of opportunistic data collection. The details of SNIP are then presented in section III. SNIP and some state-of-art mobile node-initiated probing mechanisms are modeled and compared numerically in section IV. Simulation results are then presented and analyzed in section V. Finally, section VI discusses related work and section VII concludes.

\section{Contact Probing in Opportunistic Data COLLECTION}

Figure 1 illustrates the reference network scenario of opportunistic data collection. The mobile node's mobility is uncontrollable and cannot be predicted accurately by sensor nodes. For simplicity, we assume that the network is spare enough so that at any time at most a single (static) sensor node and a single mobile node can reach each other. In the case that multiple mobile nodes move together, this assumption can be easily removed by adopting some collision avoidance techniques and allowing a sensor node to choose one of these mobile nodes randomly or based on their radio signal strength and their movement speed. We also assume that the same commodity radio (Zigbee-compilant radio, etc.) is installed on both mobile nodes and sensor nodes, i.e., they have the same communication range $(R)$. When carrying out contact probing, the radio of a sensor node is duty-cycled for achieving a long life. More specifically, the radio is turned on for a fixed period $\left(T_{o n}\right)$ and turned off for another fixed period $\left(T_{o f f}\right)$ alternatively. Hence, the duration of a cycle $\left(T_{\text {cycle }}\right)$ is the sum of $T_{o n}$ and $T_{\text {off }}$ and the duty-cycle $(d)$ equals to $T_{o n} / T_{\text {cycle }}$.

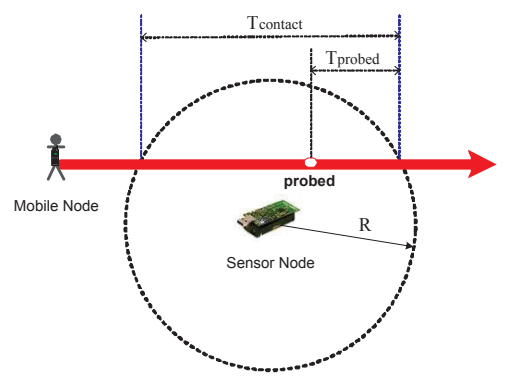

Fig. 1: Contact Probing in Opportunistic Data Collection

Under this scenario, the sensed data can be collected from a sensor node only after a mobile node approaches and they become aware of each other. As shown in figure 1, the event of the mobile node encountering a sensor node is referred to as a contact and the contact length $\left(T_{\text {contact }}\right)$ is the duration for which the mobile node stays within the communication range of the sensor node. As for $T_{\text {probed }}$, it starts immediately after both of them are aware of the presence of each other and it can be used to derive the amount of data that could be collected in this contact. For a contact probing mechanism, it should be designed so that a contact can be successfully probed with high probability and the contact is probed as early as possible. More specifically, when a sensor node's duty-cycle is fixed, a contact probing mechanism should try to maximize $\Upsilon=\frac{T_{\text {probed }}}{T_{\text {contact }}}$, the percent of contact capacity that is probed successfully for data collection.

For contact probing in opportunistic data collection, there are four processes in the system: the movement of a mobile node, the radio schedule of a mobile node, the radio schedule of a sensor node, and the beacons periodically transmitted by either mobile node or sensor node with a fixed interval $\left(T_{\text {beacon }}\right)$. To establish successful contact, a beacon must be sent out by either mobile node or sensor node when they are close to each other and their radios are both turned on. In other words, all four processes must occur at the same time. This can be difficult to achieve when mobile node's movement is uncontrollable and sensor node is required to maintain aggressive duty-cycles for reasons of longevity.

Since the mobility in opportunistic data collection is uncontrollable, a contact probing mechanism is limited to control the broadcasting of beacons and the radio schedules of mobile node and sensor node. Considering that a mobile node could have relatively abundant energy via a re-chargable battery [3][4], the radio of mobile node can be always turned on. Hence, it only needs to answer the following two questions.

1) For improving the probed contact capacity when the duty-cycle of a sensor node is fixed, who should be responsible for broadcasting the beacons?

2) For energy-efficiently probing the necessary contacts for uploading its sensor reports, how should the sensor node select the duty-cycle used by contact probing?

In this paper, we focus on the first question and leave the second one to future work.

Traditionally, a radio consumes much more energy in transmitting mode, so the resource-rich mobile node is responsible for broadcasting beacons periodically. However, these mobile node-initiated probing (MNIP) mechanisms face severe challenges in opportunistic data collection. More specifically, since a sensor node must be duty-cycled, its radio schedule is unlikely to synchronize with the beacons emanating from a mobile node. In [7], it is proposed to set $T_{o n}$ of a sensor node according to $T_{\text {beacon }}$, the interval between two consecutive beacons from the mobile node. More formally, $T_{\text {on }}=T_{\text {beacon }}+T_{p k t}$, where $T_{p k t}$ is the time needed for transmitting a packet. The authors argue that a contact will be definitely detected if a sensor node's radio is turned on during the contact. However, $T_{\text {beacon }}$ could be large in opportunistic data collection to avoid overburdening mobile node (especially when smart phones act as mobile node) and/or jamming wireless channel (even when sensor node does not exist), so $T_{\text {on }}$ must be large too, and $T_{\text {off }}$ will become huge in order to maintain a low duty-cycle. Consequently, with very high probability, a sensor node's radio will not be turned on during a contact and the contact would thus be missed. Furthermore, in opportunistic data collection, sensor nodes and mobile nodes 
may belong to different authorities and it is hard to coordinate the values of $T_{\text {beacon }}$ and $T_{\text {on }}$.

In this paper, the proposal in [7] will be referred as MNIPJOINT, and the scheme with a fixed and short $T_{\text {on }}$ will be referred as MNIP-BASIC. Both of these mechanisms will be studied and compared with SNIP.

\section{SNIP}

Due to the above shortcomings of MNIP mechanisms, SNIP, a novel sensor node-initiated probing mechanism, is proposed for improving the performance of contact probing in opportunistic data collection. In this section, the design choices of SNIP are first discussed and its details are then presented.

\section{A. Design Choices}

Our key observation is that low power radio of the mainstream sensor node platforms consumes almost the same amount of energy in transmitting and receiving/listening modes. For example, the CC2420 radio of TELOSB mote consumes $35 \mathrm{~mW}$ when transmitting at its default power level $(0 \mathrm{dBm})$ and it consumes $38 \mathrm{~mW}$ in receiving mode [4]. Hence with such a platform it is effectively free, in terms of energy usage, for a sensor node to broadcast a beacon when its radio is turned on.

Another observation is that a mobile node could be equipped with relatively abundant and rechargeable power supply and its radio used for opportunistic data collection can be always turned on. This is true even for smart phones on which opportunistic data collection is treated as a second-class task. For example, it is claimed that the talk time of Google Nexus One smart phone is 7 hours (http://www.google.com/phone/static/en_USnexusone_tech_specs.html) and the smart phone consumes about $746.8 \mathrm{~mW}$ during a voice call [3]. Considering the energy consumed by the CC2420 radio, the smart phone's battery still could last a few days even if the corresponding radio is installed and this radio is not duty-cycled. Here, we expect that Zigbee-compilant smart phones will appear with the Zigbee devices deployed for health care, smart-building, etc. Furthermore, without undermining the assumption that the mobile node's radio is always turned on for contact probing, there are still a lot of opportunities to reduce the smart phone's energy consumption based on history and/or context information. For example, a smart phone can deduce whether it is moving through accelerometer [8]. When the smart phone is static and there is no sensor node nearby, its radio for data collection can be turned off to save energy.

Based on the above observations, the radio of a mobile node is assumed to be always turned on in SNIP. In contrast with MNIP mechanisms, a sensor node in SNIP is required to broadcast a beacon immediately after its radio is turned on. Since the radio of a mobile node is always turned on, if sensor node broadcasts a beacon when they are close to each other, this contact will be definitely probed successfully, assuming of course that the beacon is not lost or corrupted due to contention, which is unlikely in sparse deployments and short range transmissions. Considering that a sensor node can turn on/off its radio relatively quickly, $T_{\text {on }}$ can be set to a small value and a sensor node can carry out contact probing frequently. Hence, a contact will be probed successfully with high probability, and the probed contact capacity will be increased significantly. Following the state transition diagrams illustrated in figure 2, the details of SNIP will be presented in the following subsection.

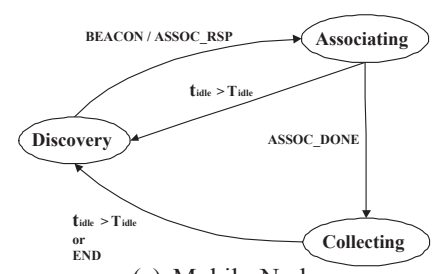

(a) Mobile Node

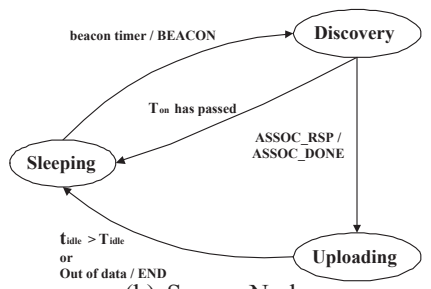

(b) Sensor Node
Fig. 2: State Transition Diagram of SNIP

\section{B. Details of SNIP}

In SNIP, a mobile node moves around in an uncontrolled manner and its radio is always turned on so that it can be discovered. After receiving a BEACON from a sensor node, a mobile node will send back ASSOC_RSP and enter into Associating state. After receiving ASSOC_DONE from sensor node, the association is complete. The mobile node will enter into Collecting state and start to collect data from the sensor node. In Collecting state, the contact may be terminated by the sensor node through sending END to the mobile node. In both Associating and Collecting states, the mobile node also keeps monitoring whether it has moved away from the sensor node. When it finds that $t_{\text {idle }}$ (the time that the channel is idle) is larger than a constant ( $\left.T_{\text {idle }}\right)$, the mobile node returns back to Discovery state and is ready to be discovered again. $T_{i d l e}$ is currently set to $50 \mathrm{~ms}$.

As for the sensor node, when its beacon/duty-cycle timer expires, it will turn on its radio, send out a BEACON, and enter into Discovery state. If it does not receive an ASSOC_RSP within $T_{o n}$, the sensor node will turn off its radio, return back Sleeping state, and start its beacon/duty-cycle timer for the next probing. $T_{o n}$ is currently set to $20 \mathrm{~ms}$, which is enough for sending a BEACON and receiving a ASSOC_RSP. If ASSOC_RSP is received in Discovery state, it will send back ASSOC_DONE, enter into Uploading state, and start to transfer data to the associated mobile node. The simple Stopand-Wait protocol is used for flow control, a retransmission timer is used for reliable data transmission, and multiple sensing reports are concatenated into one packet for reducing header overhead. If all data had been uploaded, the sensor node will send END to the mobile node for terminating this contact. In Uploading state, the sensor node also keeps monitoring whether the mobile node has moved away. When it finds that $t_{i d l e}$ is larger than $T_{i d l e}$, the sensor node will turn off its radio, return back to Sleeping state, and start its beacon/duty-cycle timer for the next probing.

\section{Analysis and Numerical Results}

In this section, we will model SNIP with a focus on the relationship between $\Upsilon$ (percent of the probed contact 
capacity), $d$ (the duty-cycle used by a sensor node during contact probing), and $T_{\text {contact }}$ (the length of a contact). More specifically, $T_{\text {probed }}$ is modeled and $\Upsilon$ can be deduced immediately $\left(\Upsilon=\frac{T_{\text {probed }}}{T_{\text {contect }}}\right)$. For comparison purpose, MNIP-BASIC and MNIP-JOINT are also modeled in the same manner, but their models are omitted in this paper due to space limitations.

\section{A. Model of SNIP}

Figure 3 shows the three processes in SNIP: the occurrence of a contact, the sensor node radio which also incorporates the beacon emanating process, and the mobile node radio.

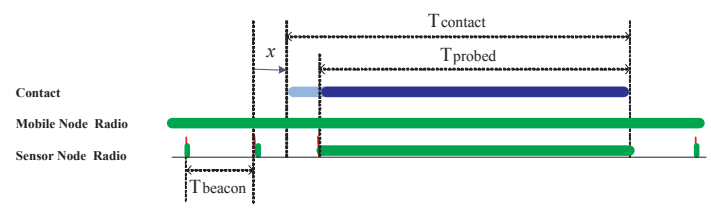

Fig. 3: Time Line of SNIP

In SNIP, sensor node will broadcast a BEACON when its radio is turned on. Hence, $T_{\text {beacon }}$, the interval between two consecutive beacons, equals to $T_{\text {cycle. }} x$ is the difference between the time that the last beacon is broadcasted and the time that a contact occurs (i.e. mobile node moves into the communication range of sensor node). Since the mobility is uncontrollable, a contact can occur at any time with the same probability. Since $T_{\text {cycle }}$ is much larger than the time needed for transmitting a BEACON, we don't consider the case that a mobile node arrives during the transmission of a BEACON. Hence, we can assume that $x$ is uniformly distributed between 0 and $T_{\text {cycle. }} T_{\text {probed }}$ can then be modeled as follow.

$$
\begin{aligned}
T_{\text {probed }}(x) & =\left\{\left(x+T_{\text {contact }}\right)-T_{\text {beacon }}\right\}^{+} \\
T_{\text {probed }} & =\frac{1}{T_{\text {cycle }}} \int_{0}^{T_{\text {cycle }}} T_{\text {probed }}(x) d x
\end{aligned}
$$

Here, $\{.\}^{+}$is defined as $\max (0,$.$) .$

\section{B. Numerical Results}

To study SNIP and compare it with MNIP-BASIC and MNIP-JOINT, the curves between $\Upsilon$ and $d$ are calculated numerically based on the above models for several typical values of $T_{\text {contact }}: 2 \mathrm{~s}, 5 \mathrm{~s}, 10 \mathrm{~s}$, and $30 \mathrm{~s}$. These values represent the time needed by a car on a freeway, a car in the city, cycling people, and walking people to pass through a distance of $50 \mathrm{~m}$, which is selected according to the communication range of current sensor node platforms. The duty-cycle of the sensor node varies from 0.001 to 0.2 .

$T_{o n}$ is set to $20 \mathrm{~ms}$ in both SNIP and MNIP-BASIC. In MNIP-JOINT, $T_{o n}$ is set to the sum of $T_{\text {beacon }}$ and $T_{p k t}$. According to the current sensor node platform, $T_{p k t}$ is set to $10 \mathrm{~ms}$. In both MNIP-BASIC and MNIP-JOINT, the evaluated values of $T_{\text {beacon }}$ are $100 \mathrm{~ms}$ and $500 \mathrm{~ms}$. The smaller values of $T_{\text {beacon }}$ are not chosen because mobile devices will be overburdened especially when smart phones are used as mobile nodes and/or the wireless channel will be jammed by beacons even when sensor node does not exist.

Figure 4 plots the numerical results of SNIP. X-axis is the duty-cycle used by a sensor node. Note that the energy

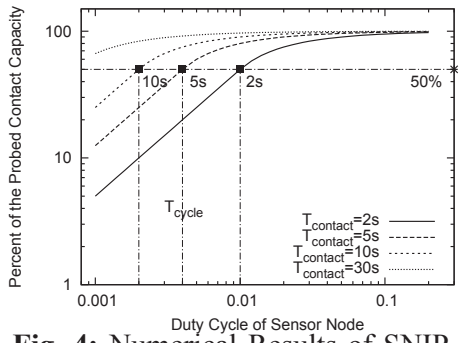

Fig. 4: Numerical Results of SNIP

consumption of a sensor node is proportional to the duty cycle and the duty-cycle of a sensor node can be used to depict the energy consumed by contact probing. Y-axis is the percent of the probed contact capacity, which determines the amount of probed contact capacity. Figure 4 indicates that $\Upsilon$ increases with $d$ and $T_{\text {contact }}$ significantly affects the curve. When a mobile node moves quickly and $T_{\text {contact }}$ is short, a sensor node needs to spend much more energy to probe the same amount of contact capacity. Figure 4 also indicates that when $T_{\text {cycle }} \geq T_{\text {contact }}, \Upsilon$ is linearly related with $d$. In fact, the closed-form equations 1 and 2 can be deduced through modeling the following two cases separately. When $T_{\text {cycle }} \geq T_{\text {contact }}$,

$$
\begin{aligned}
T_{\text {probed }} & =E\left[P_{\text {probed }}\right] * E\left[T_{\text {per_probed_contact }}\right] \\
& =\left(\frac{T_{\text {contact }}}{T_{\text {cycle }}}\right) *\left(\frac{T_{\text {contact }}}{2}\right)=\frac{T_{\text {contact }}^{2}}{2 * T_{\text {on }}} * d
\end{aligned}
$$

When $T_{\text {cycle }}<T_{\text {contact }}$,

$$
\begin{aligned}
T_{\text {probed }} & =E\left[P_{\text {probed }}\right] * E\left[T_{\text {per_probed_contact }}\right] \\
& =1 *\left(T_{\text {contact }}-\frac{T_{\text {cycle }}}{2}\right)=T_{\text {contact }}-\frac{T_{\text {on }}}{2 * d}
\end{aligned}
$$

To compare with MNIP-BASIC and MNIP-JOINT, for each value of $T_{\text {contact }}$, figure 5 plots the curves of these models together. It shows that compared with the MNIP mechanisms, SNIP probes much more contact capacity; alternatively, SNIP can achieve the same amount of probed contact capacity with much lower duty-cycle, i.e., much less energy consumption.

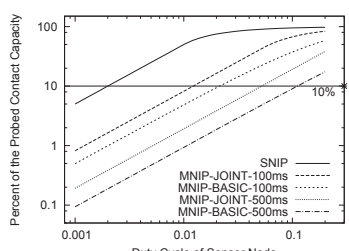

(a) $T_{\text {contact }}^{\text {Duty }}=2 \mathrm{~s}$

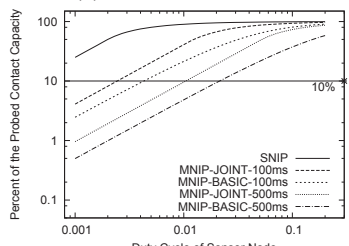

(c) $T_{\text {contact }}^{\text {Duty Cycle of Sensor Node }}=10 \mathrm{~s}$ (b) $T_{\text {contact }}^{\text {Duty }}=5 \mathrm{~s}$

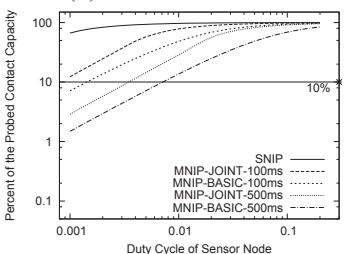

(d) $T_{\text {contact }}^{\text {Duty Cycle of Sensor Node }}=30 \mathrm{~s}$

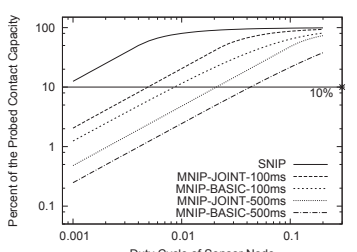

Fig. 5: Numerical Results of SNIP, MNIP-BASIC and MNIP-JOINT

\section{Simulation Results}

To evaluate SNIP in more realistic environments, SNIP is implemented in Contiki-OS [5] and extensive simulations 
are carried out in COOJA [6], which incorporates a machine code instruction level emulator of the TELOSB sensor node. For comparison, MNIP-BASIC and MNIP-JOINT are also implemented in Contiki-OS and simulated in COOJA. When implementing these contact probing mechanisms in ContikiOS, the same parameter values used in numerical analysis are adopted. For the accuracy of simulation results, in each experiment, we let a mobile node visit a sensor node repeatedly for a long time (100 hours) and assume that the sensor node always has data to be uploaded.

For validating the SNIP model, simulations are designed based on the above numerical study. The evaluated dutycycles of sensor node are $0.001,0.002,0.004,0.01,0.02,0.04$, 0.1 , and 0.2 . The evaluated values of $T_{\text {interval }}$ (the interval between two consecutive visits) are $100 \mathrm{~s}, 200 \mathrm{~s}, 500 \mathrm{~s}$, and $1000 \mathrm{~s}$. As for $T_{\text {contact }}$, its evaluated values are also $2 \mathrm{~s}, 5 \mathrm{~s}$, $10 \mathrm{~s}$, and $30 \mathrm{~s}$. When generating mobility traces, both $T_{\text {contact }}$ and $T_{\text {interval }}$ follow a normal distribution with small deviation (a tenth of the mean) or an exponential distribution. Table I lists the different combinations evaluated in this paper.

\begin{tabular}{|c||c|c|}
\hline Scenario & $T_{\text {contact }}$ & $T_{\text {interval }}$ \\
\hline \hline I & Normal Distribution & Normal Distribution \\
\hline II & Normal Distribution & Exponential Distribution \\
\hline III & Exponential Distribution & Normal Distribution \\
\hline IV & Exponential Distribution & Exponential Distribution \\
\hline
\end{tabular}

TABLE I: The Evaluated Distributions of $T_{\text {contact }}$ and $T_{\text {interval }}$

\section{A. Validation of SNIP Model}

To validate the accuracy of SNIP model, for each simulated scenario listed in table $\mathrm{I}$ and each value of $T_{\text {contact }}$, the simulation results (with different $T_{\text {interval }}$ ) and numerical results of SNIP are plotted together. Figure 6 and 7 show the plots when $T_{\text {contact }}$ equals to $2 \mathrm{~s}$ and $10 \mathrm{~s}$. For other values of $T_{\text {contact }}$, the results are similar and the plots are omitted for brevity. Figure 6 indicates that our model for SNIP is very accurate when contact length follows the normal distribution. It means that our model does capture the fundamentals of SNIP. Figure 6 also shows that $\Upsilon$ is independent of $T_{\text {interval }}$ (both the mean and the distribution followed by $T_{\text {interval }}$ ). Of course, the probed contact capacity will vary with the value of $T_{\text {interval }}$ since it determines the overall contact capacity.

However, as shown in figure 7, when contact length follows the exponential distribution, there are some differences between our model and the simulation results. $\Upsilon$ is still quite independent of $T_{\text {interval }}$, but with large variance. The simulation results are obviously better than our model when the duty-cycle of sensor node is low. The reason could be that the variance of contact length is much larger when it follows the exponential distribution. For contacts which are much longer than the mean, their $\Upsilon$ can be much larger than the value calculated based on the mean. Since these long contacts can be a significant part of the overall contact capacity, the simulation results tend to be better than the output of the model based on the mean. Hence, to accurately predict the probed contact capacity based on SNIP model, the distribution of contact length should also be considered.

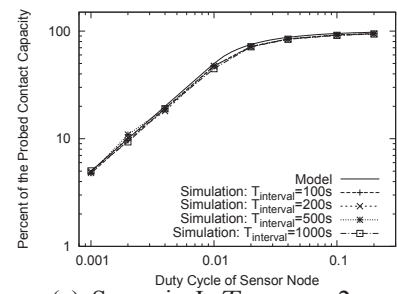

Duty Cycle of Sensor Node
(a) Scenario I: $T_{\text {contact }}=2 \mathrm{~s}$

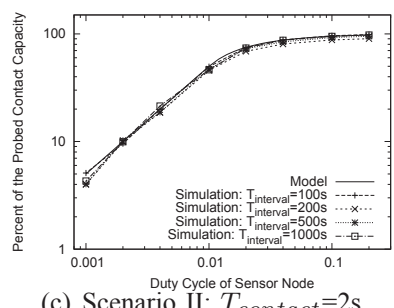

(c) Scenario II: $T_{\text {contact }}=2 \mathrm{~s}$

Fig. 6: Validation of SNIP Model: Scenario I and II

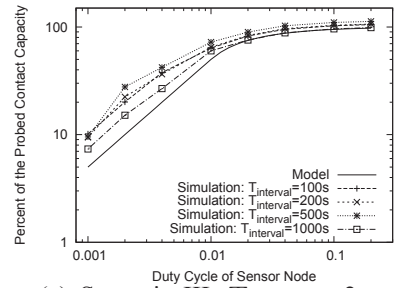

(a) Scenario III: $T_{\text {contact }}=2 \mathrm{~s}$

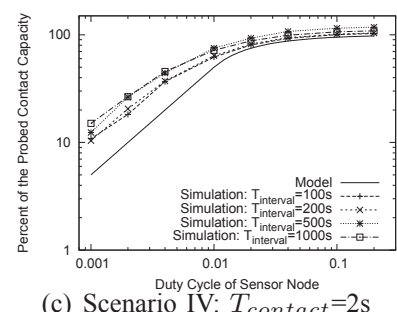

(c) Scenario IV: $T_{\text {contact }}=2 \mathrm{~s}$

Fig. 7: Validation of SNIP Model: Scenario III and IV

\section{B. Comparison of SNIP, MNIP-BASIC, and MNIP-JOINT}

To compare SNIP with MNIP-BASIC and MNIP-JOINT, simulation results of SNIP, MNIP-BASIC, and MNIP-JOINT are plotted together for each combination of the simulated scenarios and the values of $T_{\text {contact }} \& T_{\text {interval }}$. Figure 8 shows the results when $T_{\text {interval }}$ equals $200 \mathrm{~s}$ and $T_{\text {contact }}$ equals $2 \mathrm{~s}$ and $10 \mathrm{~s}$. The results with other values are similar and their plots are omitted for brevity.

Figure 8 indicates that SNIP performs much better than MNIP mechanisms in all cases, especially when the dutycycle is low. When the duty-cycle is lower than $1 \%$, compared with MNIP-JOINT with a high probing frequency ( $T_{\text {beacon }}=100 \mathrm{~ms}$ ), SNIP can improve the performance by a factor of 2-10. Hence, when duty-cycle is low, SNIP could still be much more energy efficient than MNIP mechanisms even if the transmitting mode of sensor node's radio consumes more energy than the receiving/listening mode.

\section{RELATED WORK}

Contact probing has been well studied in bluetooth-based opportunistic applications [9] and other delay-tolerant appli- 


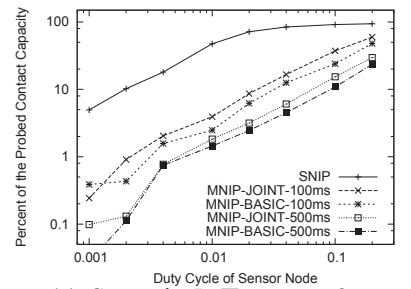

(a) Scenario I, $T_{\text {contact }}=2 \mathrm{~s}$

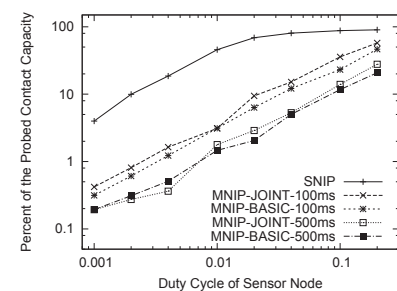

(c) Scenario II, $T_{\text {contact }}=2 \mathrm{~s}$

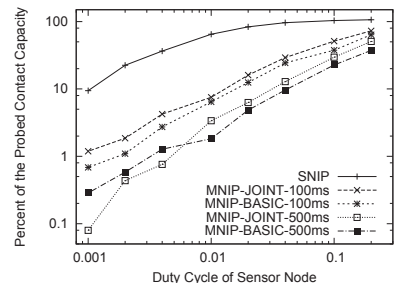

(e) Scenario III, $T_{\text {contact }}=2 \mathrm{~s}$

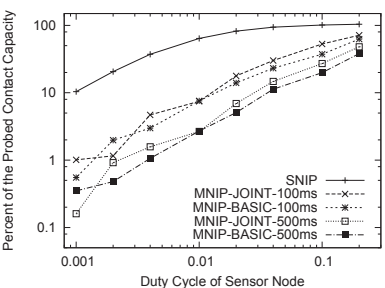

(g) Scenario IV, $T_{\text {contact }}=2 \mathrm{~s}$

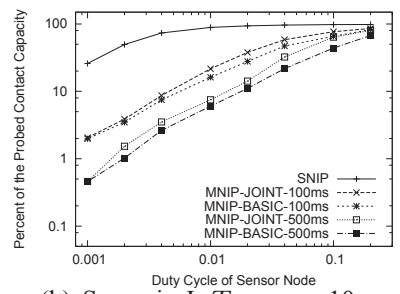

(b) Scenario I, $T_{\text {contact }}=10 \mathrm{~s}$

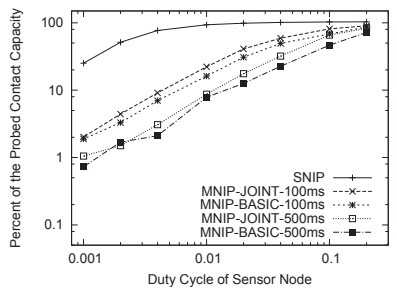

(d) Scenario II, $T_{\text {contact }}=10 \mathrm{~s}$

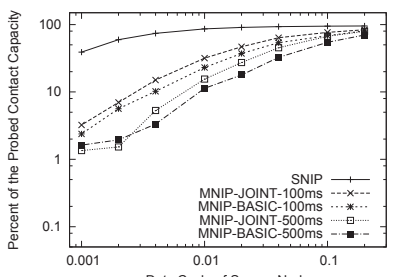

(f) Scenario III, $T_{\text {contact }}=10 \mathrm{~s}$

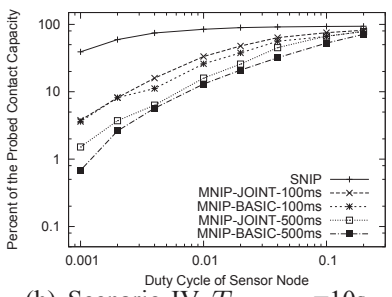

(h) Scenario IV, $T_{\text {contact }}=10 \mathrm{~s}$
Fig. 8: Simulation Results of SNIP, MNIP-BASIC, and MNIP-JOINT

cations [10]. All nodes in these applications are similar to each other and the radio consumes much more energy in transmitting mode. Through tuning the probing frequency, the proposals in [9][10] try to achieve better tradeoff between the probability of missing a contact and the energy consumed by contact probing.

In [2][11], a mobile node with controllable mobility has been used to collect data from a sensor node. The mobile node first moves to a sensor node, collects all data from this node, and moves to another sensor node. Before collecting data, a mobile node will first activate a sensor node through light, magnetic, or the second low-power radio. Hence, some additional hardware components are needed for these schemes. Low-power MAC layer protocols, such as B-MAC [12] and $\mathrm{X}-\mathrm{MAC}$ [13], have also been used for this purpose. However, in opportunistic data collection, a mobile node does not know the position of a sensor node. Hence, it cannot know when to transmit the low power preamble and cannot decide the preamble's length. In addition, the throughput of these MAC protocols is too low for opportunistic data collection with uncontrollable mobility.

In [7], mobile node-initiated probing mechanisms have also been studied in the context of opportunistic data collection. Their shortcomings have been discussed in section II. Similar to SNIP, a sensor node in Koala [14] also periodically broadcasts beacons to declare its presence. Through low power listening, the sink node can then activate the whole network hop by hop. However, the authors have not studied this scheme as a contact probing mechanism in the context of opportunistic data collection with uncontrollable mobility.

\section{CONCLUSION}

In this paper, the challenges faced by contact probing in the context of opportunistic data collection are investigated and SNIP, a Sensor Node-Initiated Probing mechanism, is proposed for improving the performance of contact probing when the duty-cycle of a sensor node is fixed. Both the analysis and simulation results indicate that SNIP performs much better than Mobile Node-Initiated Probing mechanisms, especially when the duty-cycle of a sensor node is low.

In the future, we will study how a sensor node should select the duty-cycle used by SNIP so that it can energy-efficiently probe the necessary contacts for uploading its sensor reports. We will also investigate the issues rising when smart phones act as mobile nodes, such as incentives, user privacy, and data security that are encountered in participatory sensing.

\section{ACKNOWLEDGEMENTS}

This work is supported in part by the HEA PRTLI-IV NEMBES Grant. The authors thank the Cork Constraint Computation Centre (4C) at University College Cork for use of their computing cluster.

\section{REFERENCES}

[1] R. C. Shah, S. Roy, S. Jain, and W. Brunette, "Data mules: Modeling a three-tier architecture for sparse sensor networks," in IEEE SNPA Workshop, 2003.

[2] A. A. Somasundara, A. Kansal, D. D. Jea, D. Estrin, and M. B. Srivastava, "Controllably mobile infrastructure for low energy embedded networks," IEEE Transactions on Mobile Computing, vol. 5, no. 8, pp. 958-973, august 2006.

[3] A. Carroll and G. Heiser, "An analysis of power consumption in a smartphone," in USENIX Annual Technical Conference, 2010.

[4] J. Polastre, R. Szewczyk, and D. Culler, "Telos: Enabling ultra-low power wireless research," in IPSN, 2005.

[5] A. Dunkels, B. Gronvall, and T. Voigt, "Contiki - a lightweight and flexible operating system for tiny networked sensors," in $L C N, 2004$.

[6] F. sterlind, A. Dunkels, J. Eriksson, N. Finne, and T. Voigt, "Cross-level simulation in cooja," in EWSN, 2007.

[7] G. Anastasi, M. Conti, and M. D. Francesco, "An analytical study of reliable and energy-efficient data collection in sparse sensor networks with mobile relays," in EWSN, 2009.

[8] D. H. Kim, Y. Kim, D. Estrin, and M. B. Srivastava, "Sensloc: Sensing everyday places and paths using less energy," in Sensys, 2010.

[9] C. Drula, "Fast and energy efficient neighbour discovery for opportunistic networking with bluetooth," Master Thesis, U. of Toronto, 2005.

[10] W. Wang, V. Srinivasan, and M. Motani, "Adaptive contact probing mechanisms for delay tolerant applications," in Mobicom, 2007.

[11] O. Tekdas, V. Isler, J. H. Lim, and A. Terzis, "Using mobile robots to harvest data from sensor fields," IEEE Wirelss Communications, vol. 16, no. 1, pp. 22-28, Feburary 2009.

[12] J. Polastre, J. Hill, and D. Culler, "Versatile low power media access for wireless sensor networks," in SENSYS, 2004.

[13] M. Buettner, G. V. Yee, E. Anderson, and R. Han, "X-mac: A short preamble mac protocol for duty-cycled wireless sensor networks," in SENSYS, 2006.

[14] R. Musaloiu-E, C.-J. M. Liang, and A. Terzis, "Koala: Ultra-low power data retrieval in wireless sensor networks," in IPSN, 2008. 